\title{
Cultivo de Espécies Alimentares em Quintais Urbanos de Rio Branco, Acre, Brasil
}

\author{
Amauri Siviero ${ }^{1,3}$, Thiago Andrés Delunardo ${ }^{2}$, Moacir Haverroth ${ }^{1}$, \\ Luis Cláudio de Oliveira ${ }^{1}$ e Ângela Maria Silva Mendonça ${ }^{1}$
}

Recebido em 01/03/2011. Aceito em 25/05/2011

\section{RESUMO}

(Cultivo de espécies alimentares em quintais urbanos de Rio Branco, Acre, Brasil). Os quintais são espaços de resistência no ambiente urbano que garantem a interação do homem com elementos do mundo natural. As plantas alimentares cultivadas em quintais urbanos são importantes na complementação da dieta alimentar dos moradores da cidade. Este trabalho teve como objetivo realizar um levantamento das espécies vegetais de uso alimentar cultivadas em quintais urbanos de Rio Branco. Foram realizadas entrevistas presenciais com uso de questionário específico registrando as espécies vegetais de uso alimentar, área do quintal e fatores socioeconômicos dos moradores em 132 quintais urbanos de Rio Branco entre 2009 e 2010. Os bairros selecionados para este estudo foram Aeroporto Velho, Placas e Novo Horizonte, todos situados na periferia do município. Foram detectadas 77 espécies de uso alimentar pertencentes a 34 famílias botânicas, com destaque para as famílias Solanaceae (12,6\%) e Myrtaceae (11,3\%), sendo 62,0\% de espécies frutíferas e 38,0\% de hortaliças. Do total de espécies registradas $82,3 \%$ são exóticas, sendo 34,1\% e 18,9\% associadas ainda ao uso medicinal e ornamental. Não foram identificadas associações estatísticas significativas entre a riqueza de espécies e os fatores socioeconômicos. A análise de variância não paramétrica apresentou diferenças significativas entre bairros revelando que o bairro Placas possui maior riqueza de espécies. A riqueza de espécies correlacionou positivamente com a área dos quintais. O cultivo das plantas alimentares em quintais urbanos de Rio Branco auxilia no tratamento de doenças e promove a conservação da agrobiodiversidade, bem estar aos moradores pela melhoria da paisagem, ambiência microclimática e espaço de lazer.

Palavras-chave: agrobioversidade, Amazônia, agricultura urbana

\begin{abstract}
(Cultivation of food species in urban gardens in Rio Branco, Acre, Brazil). Home gardens are urban spaces that guarantee human interaction with elements from the natural word, and the cultivation of food plants in these gardens can be important in complementing the diet of city residents. The goal of this work was to make an assessment of the eatable food plants grown in home gardens in the city of Rio Branco. In 2009 and 2010, on-site interviews were carried out using a questionnaire, which recorded the plant species of each kind of food, area of the gardens and the socioeconomic factors, for 132 gardens in Rio Branco. The neighborhoods selected for this study, Aeroporto Velho, Novo Horizonte and Placas, were located in the outskirts of the city. Seventy-seven food plants were recorded, which belong to 34 plant families. Of these, Solanaceae (12.6\%) and Myrtaceae (11.3\%) with $62.0 \%$ of the kinds of fruits and $38.0 \%$ of the vegetables. Of the total number of plants recorded, $82.3 \%$ were exotic, $34.1 \%$ were medicinal and $18.9 \%$ were ornamental. No significant statistical association was detected between species and socioeconomic factors. Nonparametric analysis of variance indicated significant differences between neighborhoods, showing that Placas had a higher quantity of species. The quantity of species correlated positively in the home gardens. Food plants cultivated in Rio Branco gardens conserve agrobiodiversity and aid in the health and well-being of the residents by improving the landscape, ambience and leisure space of the city.
\end{abstract}

Key words: agrobiodiversity, Amazon, urban agriculture

\footnotetext{
1 Empresa Brasileira de Pesquisa Agropecuária, Centro de Pesquisa Agroflorestal do Acre, Rio Branco, AC, Brasil

2 Universidade Federal do Acre, Rio Branco, AC, Brasil

3 Autor para correspondência: asiviero@cpafac.embrapa.br
} 


\section{Introdução}

A produção de alimentos sempre esteve associada às áreas rurais, restando aos centros urbanos o papel de grandes consumidores de produtos agrícolas produzidos no campo. No entanto, a cada dia se observa um aumento no uso agrícola da área nos quintais domésticos e hortas comunitárias urbanas, notadamente nas zonas periféricas das grandes e médias cidades, para a produção de alimentos.

Para Kumar \& Nair (2004) o quintal é uma unidade de paisagem onde ocorre um elevado número de espécies e as interações estabelecidas com os respectivos idealizadores satisfazem necessidades econômicas, sociais e culturais específicas do grupo envolvido. Os quintais são espaços de fácil acesso para os moradores cultivarem uma diversidade de espécies e funções como: estética, lazer, alimentação e medicinal. Interessante observar que em todas as regiões tropicais do mundo ocorre o sistema agroflorestal denominado de quintal, com suas variantes em cada região ou país, sendo muito semelhantes na sua estrutura e função.

Segundo Amorozo (2002) o quintal se refere ao espaço do terreno situado ao redor da casa regularmente manejado, onde são cultivadas plantas e também são criados animais domésticos de pequeno porte. Uma alta diversidade de espécies é cultivada nos quintais urbanos, periurbanos e agroflorestais, com múltiplas finalidades de uso artesanal, ornamental, paisagístico, além de proporcionar melhoria do microclima (sombra), fonte de fibra, uso mágico e, notadamente, plantas de uso alimentar e medicinal (Nair 2004).

Estudos realizados em quintais urbanos e rurais de vários países reportam altos níveis de diversidade genética de plantas inter e intra-específica, notadamente, de variedades de culturas tradicionais e crioulas que estão sendo conservadas nestes ambientes (Galluzzi et al. 2010). O cultivo de espécies vegetais em quintais auxilia na complementação da dieta, gerando maior segurança alimentar, pois oferece alimentos seguros e de fácil acesso em quantidade e qualidade. O impacto positivo da agricultura urbana na situação nutricional das famílias tem sido avaliado no Brasil e em vários países do mundo (Aquino \& Assis 2007).

Os estudos sobre desenvolvimento local freqüentemente se concentram na economia de fluxos econômicos formais. No entanto, a economia informal é um importante fator de sobrevivência, notadamente para as classes de renda mais baixa da população brasileira. Os quintais urbanos na Amazônia representam um tipo de estratégia de sobrevivência e de resistência dos moradores urbanos pobres situados nas periferias das capitais da Amazônia (Slinger 2000). A manutenção de hortas caseiras em grandes centros ajuda os moradores a sobreviver em uma cidade mais urbanizada, fornecendo alimentos diretamente através dos quintais ou via trocas com vizinhos e parentes. Os vínculos sociais baseados na economia informal de trocas de mercadorias, experiências e espécies vegetais são fortalecidos nas cidades da Amazônia (Winklerprins \& Sousa 2005).
Os alimentos produzidos na própria residência significam, freqüentemente, uma redução importante nos gastos com alimentação. As famílias pobres urbanas podem gastar de 60 a $80 \%$ de sua renda com comida. A produção de alimentos em casa torna a pequena renda das pessoas disponível para outras despesas e, além disso, o melhor acesso à comida e à renda afeta positivamente a capacidade das pessoas para trabalhar e investir (Singer 2002).

Os quintais urbanos podem ser considerados como sistemas agroflorestais que desempenham função ecológica, conservam alta diversidade de plantas na sua composição, asseguram variabilidade genética, constituindo importantes bancos de germoplasma, representando sistemas sustentáveis com maior resistência a doenças, pragas e adaptabilidade (Amaral \& Guarim Neto 2008), principalmente na Amazônia (Ferreira \& Pires Sablayrolles 2009).

Os quintais urbanos na Amazônia representam um tipo de estratégia de sobrevivência e de resistência dos moradores urbanos pobres situados nas periferias das capitais da Amazônia (Slinger 2000). A manutenção de hortas caseiras em grandes centros ajuda os moradores a sobreviver em uma cidade mais urbanizada, fornecendo alimentos diretamente através dos quintais ou via trocas com vizinhos e parentes. Os vínculos sociais baseados na economia informal de trocas de mercadorias, experiências e espécies vegetais são fortalecidos nas cidades da Amazônia (Winklerprins \& Sousa 2005).

Emperaire \& Eloy (2008) relataram o fenômeno do estreitamento da relação entre comunidades florestais e áreas urbanas na Amazônia. De acordo com as autoras, as atividades de produção agrícola, originalmente praticadas na floresta, estão sendo modeladas na periferia das cidades, construindo um novo mosaico agrícola urbano. Estudos realizados em quintais urbanos e não urbanos no Peru indicam que a diversidade dos quintais é fortemente relacionada às características específicas de tamanho e forma do local, característica socioeconômicas e de acesso a material de plantio, como a disponibilidade de sementes e mudas (Coomes \& Ban 2004).

No caso específico do Acre tem sido verificado um intenso processo de êxodo rural, notadamente para a capital do estado, Rio Branco provocou a fundação de diversos bairros que apresentam uma população composta basicamente de ex-seringueiros, de baixo poder aquisitivo e com grande conhecimento empírico acumulado sobre o cultivo de espécies alimentares ao redor da casa (Oliveira 1982; Schmink \& Cordeiro 2008).

A literatura acerca da riqueza e uso de espécies alimentares cultivadas em quintais urbanos de Rio Branco acusa apenas o trabalho desenvolvido por Delunardo (2008). Desta forma, o objetivo deste trabalho foi identificar as espécies vegetais de uso alimentar cultivadas em quintais urbanos de Rio Branco, uma vez que boa parte dos seus moradores são detentores de saber tradicional a respeito do uso dos recursos vegetais da floresta, principalmente das plantas alimentares. 


\section{Material e métodos}

\section{Área de estudo}

A cidade de Rio Branco está situada na extremidade da Amazônia Ocidental e exerce forte atração populacional, com alta taxa de urbanização, recebendo uma população oscilante e altamente diversa do interior do Acre e de outras regiões do país. O município de Rio Branco tem uma população de, aproximadamente, 320.000 habitantes apresentando cerca de 360 bairros, conjuntos habitacionais (COHAB), loteamentos e invasões de diversos tamanhos incluindo as áreas rurais ocupando uma área de 883.143,74 ha. A cidade está dividida em sete zonas regionais municipais distribuídas em dois grandes distritos (Schmink \& Cordeiro 2008).

O clima de Rio Branco é do tipo Am, ou seja, quente e úmido, apresentando uma estação chuvosa com altos índices pluviométricos de outubro a março e a precipitação anual varia de 2000 a $2100 \mathrm{~mm}$. A vegetação do município de Rio Branco é atualmente classificada como Área Antrópica (AP). No entanto, a vegetação original foi classificada como Floresta Tropical Aberta (Acre 2006). Os solos de Rio Branco são de origem sedimentar apresentando desde solos bem desenvolvidos, como os latossolos, até solos jovens, como os neossolos, destacando-se os argissolos, plintossolos, luvissolos e gleissolos com ocorrência de terras baixas de relevo ondulado. O município apresenta variação altimétrica de 130 a 330 metros de altitude no extremo sudoeste (Rio Branco 2007) (Figura 1).
A primeira etapa do estudo consistiu na realização de visita a diversos órgãos da Prefeitura Municipal de Rio Branco visando obter informações sobre o processo de formação dos bairros da cidade para auxiliar na melhor escolha dos locais. Posteriormente, se deu a escolha dos bairros para a pesquisa usando os seguintes critérios: a. abranger bairros localizados em diferentes zonas regionais; bairros que abrigam moradores de baixa renda localizados, notadamente, na periferia da cidade com igual eqüidistância do centro e entre si, onde as plantas cultivadas no quintal são mais importantes para a segurança alimentar da família; b. bairros periféricos ao centro, onde ocorrem quintais maiores visando aumentar a possibilidade de entrevistar maior número de ex-extrativistas nativos e d. apresentar épocas de fundação distintas propiciando o acesso às moradias construídas nos vários movimentos de migração do campo para a cidade. As informações detalhadas que subsidiaram a seleção dos bairros para a pesquisa foram obtidas junto ao Serviço de Engenharia e Arquivo da Prefeitura Municipal de Rio Branco e consultas na obra de Schmink \& Cordeiro (2008).

Desta forma foram escolhidos para esta pesquisa três bairros: O primeiro foi o bairro Aeroporto Velho, fundado em 1960, localizado na zona Sul da capital pertencente à regional VI de Rio Branco, formado por 16 loteamentos menores e abriga cerca de 80.000 pessoas. O segundo foi o das Placas tem sua origem em 1965 e está localizado em na regional III da cidade, de Rio Branco sendo formado por 30 loteamentos, invasões e pequenos conjuntos habitacionais. O último foi o bairro Novo Horizonte, criado em 1978, sen-

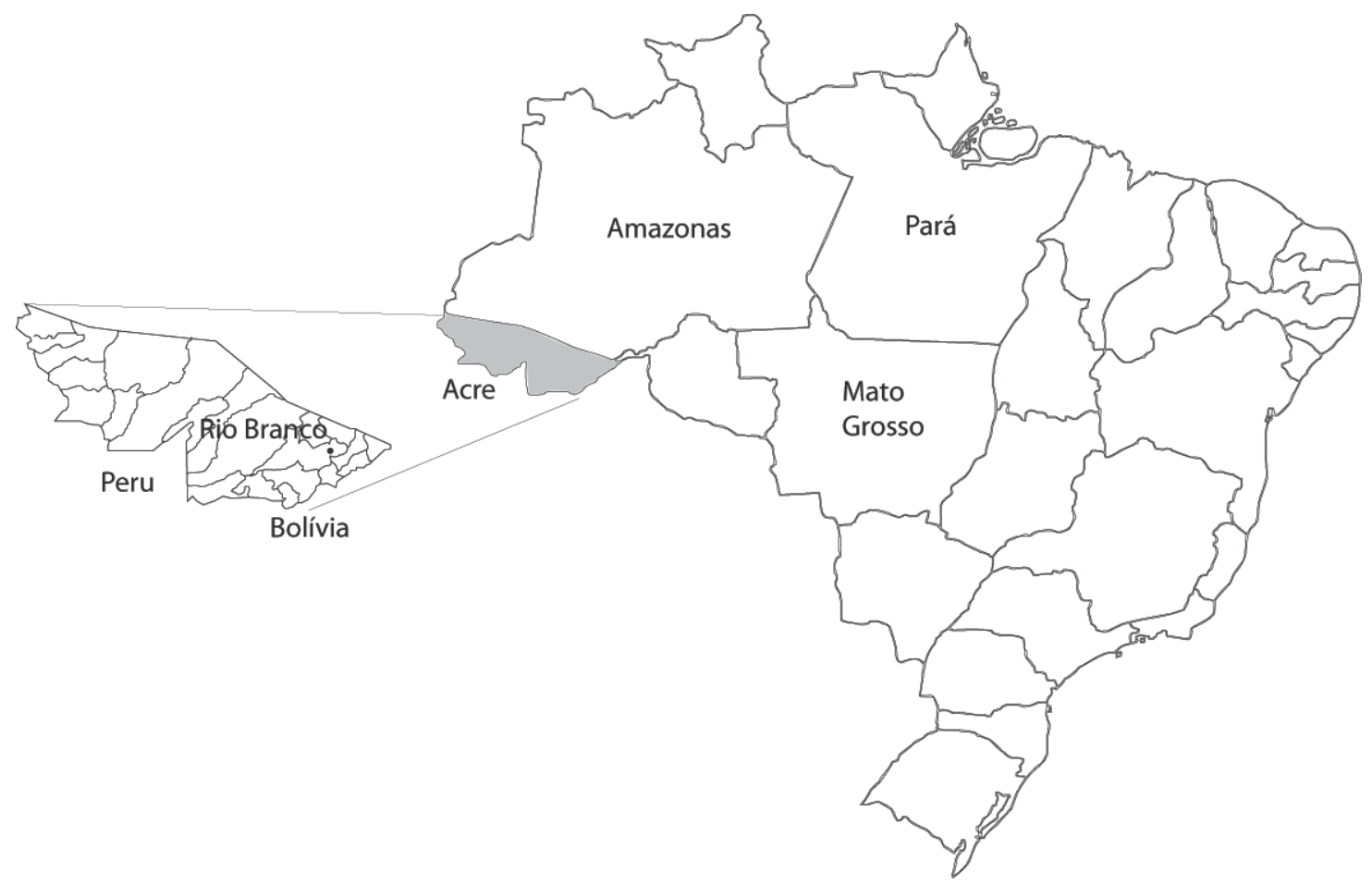

Figura 1. Localização da área de estudo com indicação da cidade de Rio Branco, capital do Acre 
do composto por 13 quadras (quarteirões) foi e está situado na zona oeste em relação à área central de Rio Branco. O bairro pertence à regional $\mathrm{V}$ (Floresta) que é composta por 34 pequenos conjuntos habitacionais e loteamentos. Em seguida houve contato com as associações de moradores de cada bairro, visando uma melhor aproximação da comunidade local e explicando a natureza e os objetivos da pesquisa.

\section{Amostragem e coleta de dados}

A escolha das residências em cada bairro foi feita pelo método de amostragem sistemática de pontos de amostragem, partindo da escolha de uma quadra central do bairro, afastado de vias de grande circulação de veículos. Uma vez escolhida, aleatoriamente, a primeira residência da quadra para entrevista, o próximo ponto de amostragem escolhido eram as duas casas situadas após o primeiro ponto e assim sucessivamente, circundando todo o perímetro da quadra. Este trabalho foi auxiliado com uso de mapas cartográficos e imagens de satélite.

Ao todo foram visitadas 132 residências nos bairros Aeroporto Velhas (53), Placas (44) e Novo Horizonte (35) sendo selecionadas segundo os critérios acima mencionados e pela curva de acumulação de espécies (Delunardo, 2008). A metodologia de coleta de dados primários foi feita via abordagem qualitativa utilizando técnicas de entrevista semi estruturada, seguindo um roteiro-guia padrão e de observação direta participante (Albuquerque \& Lucena 2004.). O entrevistado foi o membro designado pela família no momento do primeiro contato estabelecido pelo entrevistador. Cada entrevista foi realizada após a assinatura pelo entrevistado de um termo de consentimento aceitando participar da pesquisa e autorizando a divulgação dos resultados do estudo.

Foram pesquisados aspectos qualitativos e quantitativos da dinâmica estrutural e espacial dos quintais e socioeconômicos dos entrevistados. A entrevista aplicada considerou os aspectos levantados por Millat-e-Mustafa (1998) e constou de questões sobre a área do quintal e dados das espécies alimentares cultivadas como: nome comum, forma de obtenção da planta (floresta, vizinho, etc.), indicação de usos da espécie, hábito de crescimento, propagação e formas de consumo.

As espécies vegetais mais comuns de cada quintal foram contabilizadas em campo. As espécies que não puderam ser identificadas no local foram encaminhadas para o Herbário da Universidade Federal do Acre visando à identificação botânica em nível de família, gênero e espécie. A identificação das plantas foi feita por comparação com exsicatas do herbário, chaves de identificação, levantamento bibliográfico em literatura especializada e buscas em bases de dados como o MOBOT (MOBOT 2010). A busca realizada junto ao sítio da rede mundial de computadores MOBOT foi também útil na obtenção da informação sobre a provável origem geográfica das espécies. O sistema taxonômico adotado neste trabalho foi o APG III (The Angiosperm Phylogeny
Group 2009). A nomenclatura dos nomes científicos foi conferida utilizando as bases de dados Plantminer (Carvalho et al. 2010).

Análise de dados - Todas as análises foram realizadas no programa estatístico Statistical Analysis System, versão 9.1 (SAS 2003). Para determinar a distribuição da variável riqueza de espécies foi utilizado o procedimento PROC UNIVARIATE NORMAL e seus valores entre variáveis foram comparados através de análise de variância não paramétrica (PROC NPAR1WAY). As associações não paramétricas entre as variáveis quantitativas, riqueza, idade e tempo de moradia foram testadas por meio do PROC CORR Spearman (análise de correlação). Para determinar a associação entre as variáveis qualitativas sexo, escolaridade, estado civil e naturalidade com a riqueza de espécies, foram utilizadas tabelas de contingência (PROC FREQ).

\section{Resultados e discussão}

A área média dos quintais foi de $397,9 \mathrm{~m}^{2}$ para o bairro Placas, $84,25 \mathrm{~m}^{2}$ para Novo Horizonte e $169,6 \mathrm{~m}^{2}$ para Aeroporto Velho, e a área de utilização efetiva da residência como quintal foi de $82 \%, 44 \%$ e $62 \%$ respectivamente. Os resultados indicaram uma grande riqueza de plantas alimentares nas residências, registrando-se 77 espécies de plantas de uso alimentar, 57 gêneros, pertencentes a 34 famílias botânicas distintas, com destaque para as famílias Solanaceae (12,6\%) e Myrtaceae (11,3\%) (Tabela 1).

Entre as plantas alimentares mais cultivadas merece destaque as espécies frutíferas (62,0\%) e hortaliças (38\%), sendo as mais comuns "coco" (Cocos nucifera L.), citros - "laranja", "tangerina" e "limão" (Citrus spp.), "cupuaçu" (Theobroma grandiflorum (Willd. ex Spreng.) K. Schum.) e a "acerola" (Malpighia glabra DC.). Entre as espécies hortícolas merece destaque a "cebola de palha" (Allium schoenoprasum L.) e o "quiabo" (Abelmoschus esculentus (L.) Moench). O consumo fresco $(67 \%)$ e na forma de sucos $(25,6 \%)$ foram os principais modos consumo das plantas.

Quanto ao hábito de crescimento das plantas alimentares tem-se: arbóreo $(38,0 \%)$, arbustivo $(31,6 \%)$, herbáceo $(27,8 \%)$ e do tipo cipó $(2,6 \%)$. Estes valores indicam que todos os estratos da vegetação dos quintais são utilizados para o cultivo de plantas alimentares. As espécies estão dispostas em diferentes estratos nos quintais e cada nível do estrato vegetal parece estar preenchido de espécies com funções de modo a compor a paisagem ou da utilidade das espécies pelos moradores. Desta forma cada quintal é um produto da percepção da arquitetura paisagística daquele que o maneja, associado ao espaço disponível para o cultivo.

Durante a presente pesquisa verificou-se que os entrevistados têm o costume de cultivar hortaliças (folhosas e condimentares) em canteiros suspensos e separados, uma vez que essas plantas exigem maiores cuidados quanto ao solo, geralmente mal drenado. Esta técnica aumenta a luminosidade para as plantas, protege dos animais domésticos e facilita 
Tabela 1. Espécies alimentares de quintais urbanos de Rio Branco, capital do Acre.

\begin{tabular}{|c|c|c|c|c|c|}
\hline Família & Nome comum & Nome científico & Outros usos & Hábito & Origem \\
\hline \multirow{3}{*}{ Anacardiaceae } & Caju & Anacardium ocidentale L. & Med & Erv & $\mathrm{N}$ \\
\hline & Manga & Mangifera indica L. & Med & Av & $\mathrm{E}$ \\
\hline & Cajá & Spondias mombin $\mathrm{L}$. & Med & $\mathrm{Av}$ & $\mathrm{E}$ \\
\hline \multirow{4}{*}{ Annonaceae } & Graviola & Annona muricata L. & Med & $\mathrm{Av}$ & $\mathrm{E}$ \\
\hline & Biribá & Rollinia mucosa (Jacq.) Baill. & - & $\mathrm{Av}$ & $\mathrm{E}$ \\
\hline & Pinha & Annona sp & Orn & $\mathrm{Av}$ & $\mathrm{E}$ \\
\hline & Ata & Annona squamosa L. & & Av & $\mathrm{E}$ \\
\hline \multirow{4}{*}{ Apiaceae } & Chicória da Amazônia & Erygium foetidum L. & Med & Erv & $\mathrm{E}$ \\
\hline & Coentro & Coriandrum sativum $\mathrm{L}$. & - & Erv & $\mathrm{E}$ \\
\hline & Erva doce & Foeniculum vulgare Mill. & - & Erv & $\mathrm{E}$ \\
\hline & Salsa & Petroselinum crispum $\mathrm{L}$. & - & Erv & $\mathrm{E}$ \\
\hline \multirow{2}{*}{ Araceae } & Inhame & Colocasia $\mathrm{sp}$ & - & Erv & $\mathrm{E}$ \\
\hline & Taioba & Xanthosoma sagittifolium Schoot & - & Erv & $\mathrm{E}$ \\
\hline \multirow{6}{*}{ Arecaceae } & Buriti & Mauritia flexuosa $\mathrm{L}$ & Orn & $\mathrm{Av}$ & $\mathrm{N}$ \\
\hline & Açaí solteiro & Euterpe precatoria Mart. & Med/ Orn & $\mathrm{Av}$ & $\mathrm{N}$ \\
\hline & Açaí touceira & Euterpe oleraceae Mart. & Med/ Orn & $\mathrm{Av}$ & $\mathrm{N}$ \\
\hline & Coco & Cocos nucifera $\mathrm{L}$. & Orn & $\mathrm{Av}$ & $\mathrm{E}$ \\
\hline & Pupunha & Bactris gasipaes Kunth & Orn & $\mathrm{Av}$ & $\mathrm{N}$ \\
\hline & Bacaba & Oenocarpus bacaba Mart. & Orn & $\mathrm{Av}$ & $\mathrm{N}$ \\
\hline \multirow{3}{*}{ Brassicaceae } & Couve & Brassica oleracea L. var. acephala DC. & Med & Erv & $\mathrm{E}$ \\
\hline & Agrião & Rorippa nasturtium-aquaticum (L.) Hayek & - & Erv & $\mathrm{E}$ \\
\hline & Mostarda & Brassica juncea (L.) Coss. & - & Erv & $\mathrm{E}$ \\
\hline Bromeliaceae & Abacaxi & Ananas comosus (L.) Merril. & Orn & Erv & $\mathrm{E}$ \\
\hline Caricaceae & Mamão & Carica papaya $\mathrm{L}$ & Med & $\mathrm{Av}$ & $\mathrm{E}$ \\
\hline Convolvulaceae & Batata Doce & Ipomoea batatas (L.) Lam. & - & Erv & $E$ \\
\hline \multirow[t]{2}{*}{ Cucurbitaceae } & Abóbora & Cucurbita pepo L. & - & Erv & $\mathrm{E}$ \\
\hline & Melão & Cucumis melo L. & - & Erv & $\mathrm{E}$ \\
\hline Dioscoreaceae & Cará & Dioscorea bulbifera L. & - & Erv & $\mathrm{E}$ \\
\hline Euphorbiaceae & Macaxeira & Manihot esculenta Crantz & - & Arb & $\mathrm{N}$ \\
\hline Fabaceae & Feijão & Phaseolus vulgaris $\mathrm{L}$. & - & Erv & $E$ \\
\hline Lamiaceae & Manjericão roxo e branco & Ocimum sp. & Med & Erv & $\mathrm{E}$ \\
\hline Lauraceae & Abacate & Persea americana Mill. & Med & $\mathrm{Av}$ & $\mathrm{E}$ \\
\hline \multirow{2}{*}{ Liliaceae } & Cebolinha & Allium schoenoprasum L. & - & Erv & $\mathrm{E}$ \\
\hline & Alho & Allium sativum $\mathrm{L}$. & Med & Erv & $\mathrm{E}$ \\
\hline Malpighiaceae & Acerola & Malpighia glabra DC. & Med & Arb & $\mathrm{E}$ \\
\hline Malvaceae & Quiabo & Abelmoschus esculentus (L.) Moench & - & Arb & $\mathrm{E}$ \\
\hline \multirow{2}{*}{ Moraceae } & Amora & Morus spp. & Med & $\mathrm{Av}$ & $\mathrm{E}$ \\
\hline & Jaca & Artocarpus heterophyllus Lam. & - & $\mathrm{Av}$ & $\mathrm{E}$ \\
\hline Musaceae & $\begin{array}{l}\text { Banana (maçã, prata e } \\
\text { comprida) }\end{array}$ & Musa spp. & Orn & Abr & $\mathrm{E}$ \\
\hline \multirow{9}{*}{ Myrtaceae } & Araçá boi & Eugenia stipitata Lim. & - & Arb & $\mathrm{N}$ \\
\hline & Goiaba & Psidium guajava L. & Med & $\mathrm{Av}$ & $\mathrm{N}$ \\
\hline & Pitanga & Eugenia uniflora L. & Orn & Arb & $\mathrm{N}$ \\
\hline & Araçá pera & Psidium acutangulum DC. & Orn & Arb & $\mathrm{N}$ \\
\hline & Uvaia & Eugenia pyriformis Cambess. & - & Av & $\mathrm{N}$ \\
\hline & Araçazinho & Psidium sartorianum (Berg) Niedenzu & - & Arb & $\mathrm{N}$ \\
\hline & Jabuticaba & Plinia trunciflora (O. Berg) Kausel & Orn & $\mathrm{Av}$ & $\mathrm{N}$ \\
\hline & Jambo & Eugenia malaccensis $\mathrm{L}$. & Orn & $\mathrm{Av}$ & $\mathrm{E}$ \\
\hline & Jamelão (azeitona) & Syzygium jambolanum DC. & - & $\mathrm{Av}$ & $\mathrm{E}$ \\
\hline Oxalidaceae & Carambola & Averrhoa carambola L. & Med & $\mathrm{Av}$ & $\mathrm{E}$ \\
\hline Passifloraceae & Maracujá & Passiflora edulis Sims & Med & Trep & $\mathrm{N}$ \\
\hline Pedaliaceae & Gergelim & Sesamum indicum $\mathrm{L}$. & - & Arb & $\mathrm{E}$ \\
\hline
\end{tabular}


Tabela 1. Continuação.

\begin{tabular}{|c|c|c|c|c|c|}
\hline Família & Nome comum & Nome científico & Outros usos & Hábito & Origem \\
\hline \multirow{3}{*}{ Poaceae } & Cana & Saccharum officinarum L. & - & Arb & $\mathrm{E}$ \\
\hline & Milho & Zea mays L. & - & Arb & $\mathrm{E}$ \\
\hline & Sorgo & Sorghum sp & - & Arb & $\mathrm{E}$ \\
\hline Punicaceae & Romã & Punica granatum $\mathrm{L}$. & Med & Arb & $\mathrm{E}$ \\
\hline Rosaceae & Morango & Fragaria vesca $\mathrm{L}$. & Med & Erv & $E$ \\
\hline \multirow{2}{*}{ Rubiaceae } & Noni & Morinda citrifolia $\mathrm{L}$. & Med & Arb & $\mathrm{E}$ \\
\hline & Café & Coffea spp. & - & Arb & $\mathrm{E}$ \\
\hline \multirow{3}{*}{ Rutaceae } & Limão & Citrus limon (L.) Burm. & Med & $\mathrm{Av}$ & $E$ \\
\hline & Tangerina & Citrus reticulata Blanco & - & Av & $\mathrm{E}$ \\
\hline & Laranja & Citrus spp. & Med & $\mathrm{Av}$ & $\mathrm{E}$ \\
\hline Sapotaceae & Abiu & Pouteria caimito (Ruiz \& Pav.) Radlk. & - & $\mathrm{Av}$ & $\mathrm{N}$ \\
\hline \multirow{10}{*}{ Solanaceae } & Pimenta & Capsicum sp. & - & Arb & $\mathrm{N}$ \\
\hline & Pimentão & Capsicum annuum $\mathrm{L}$. & - & Arb & $\mathrm{N}$ \\
\hline & Pimenta malagueta & Capsicum frutescens $\mathrm{L}$. & - & Arb & $\mathrm{N}$ \\
\hline & Pimenta de cheiro & Capsicum chinense Jacq. & - & Arb & $\mathrm{N}$ \\
\hline & Cubiu & Solanum sessiliflorum Dunal. & Med & Arb & $\mathrm{N}$ \\
\hline & Tomate & Solanum lycopersicum $\mathrm{L}$. & - & Arb & $\mathrm{E}$ \\
\hline & Tomate cereja & Solanum pimpinellifolium $\mathrm{L}$. & - & Arb & $\mathrm{E}$ \\
\hline & Batata doce & Solanum tuberosum $\mathrm{L}$. & - & Erv & $\mathrm{E}$ \\
\hline & Pimenta olho de peixe & Capsicum chinense Jacq. & - & Arb & $\mathrm{E}$ \\
\hline & Jiló & Solanum jilo Raddi. & - & Arb & $\mathrm{E}$ \\
\hline \multirow{3}{*}{ Sterculiaceae } & Cacau & Theobroma cacao $\mathrm{L}$. & Orn & $\mathrm{Av}$ & $\mathrm{E}$ \\
\hline & Cupuaçu & Theobroma grandiflorum (Willd. ex Spreng.) K. Schum. & Med & Av & $\mathrm{N}$ \\
\hline & Cacau da mata & Theobroma spp. & Orn & $\mathrm{Av}$ & $\mathrm{N}$ \\
\hline Vitaceae & Uva & Vitis sp & - & Trep & $\mathrm{E}$ \\
\hline \multirow{2}{*}{ Zingiberaceae } & Gengibre & Zingiber officinale Roscoe & Med & Arb & E \\
\hline & Açafrão & Curcuma longa $\mathrm{L}$. & - & Arb & $\mathrm{E}$ \\
\hline
\end{tabular}

Legenda: Orn = ornamental, $\mathrm{Med}=$ medicinal, $\mathrm{N}$ = nativa, $\mathrm{E}=$ exótica. Trep = trepadeira, Av = árvore, Arb = arbusto, erv = erva.

o manejo e o controle de doenças e pragas. A freqüência de plantas de uso alimentar e medicinais de quintais urbanos e rurais ocorre, geralmente, em números equivalentes (Amaral \& Guarim Neto 2008). Amorozo (2002) relatou que em cidades mais industrializadas a freqüência de ocorrência de espécies de uso ornamental é notadamente mais elevada quando comparadas as outras categorias.

Nesta pesquisa foi encontrado um grande número de espécies exóticas sendo cultivadas nos quintais (68\%), provavelmente, devido cerca de metade dos moradores serem oriundos de cidades fora da Amazônia e ao intercâmbio de mudas e sementes de espécies frutíferas e hortaliças entre parentes e vizinhos de outras regiões conforme reportado por Eichemberg et al. (2009) e Carniello et al. (2010). Das espécies alimentares identificadas nos quintais de Rio Branco $28,9 \%$ são também de uso medicinal e 16,8\% apresentam uso ornamental.

Semedo \& Barbosa (2007) relataram que a ocorrência de frutíferas em quintais caseiros de Boa Vista-RR segue um padrão concentrado na escolha de poucas espécies, não-originárias da Amazônia, mas tradicionalmente consagradas por seu uso na produção de frutos como: Theobroma grandiflorum, seguido pelas exóticas, como, Cocos nucifera, Citrus spp., Musa sp., Mangifera indica e Eugenia malaccensi.
O resultado do estudo socioeconômico dos entrevistados revelou que a maioria dos responsáveis pela condução e manejo do quintal é do gênero feminino e casado. Cerca de $60 \%$ dos entrevistados apresentam baixa escolaridade. Os aposentados e as donas de casa foram as principais categorias de ocupação dos moradores. Quanto à naturalidade dos entrevistados, constatou-se que a maioria (79 a 88\%) nasceu no Estado do Acre, dos quais 55\% são oriundos de cidades do interior do estado. Os que moram em Aeroporto Velho são mais idosos e moram há mais tempo no bairro (Tabela 2).

Embora a grande maioria dos entrevistados declarasse ter nascido no Acre é importante salientar que boa parte dos seus genitores é constituída de imigrantes que vieram do nordeste do Brasil, notadamente, do estado do Ceará, na qualidade de soldados da borracha, por ocasião da primeira e segunda guerra (Oliveira. 1982).

Analisando-se os resultados extraídos das tabelas de contingência observa-se que não foram encontradas associações significativas entre riqueza de plantas alimentares e as variáveis qualitativas: sexo $\left(\mathrm{gl}=20 ; \mathrm{p}>\chi^{2}=0,6926\right)$, escolaridade ( $\left.\mathrm{gl}=60 ; \mathrm{p}>\chi^{2}=0,0925\right)$, naturalidade ( $\mathrm{gl}=$ 40; $\left.\mathrm{p}>\chi^{2}=0,6638\right)$ e estado civil $\left(\mathrm{gl}=20 ; \mathrm{p}>\chi^{2}=0,8548\right)$. Os resultados da análise de correlação revelaram que não 
Tabela 2. Dados socioeconômicos dos moradores de três bairros de Rio Branco, capital do Acre.

\begin{tabular}{|c|c|c|c|c|}
\hline Dados socioeconômicos & Categorias & Placas & Aeroporto Velho & Novo Horizonte \\
\hline \multirow{2}{*}{ Gênero (\%) } & Masculino & 23 & 31 & 32 \\
\hline & Feminino & 77 & 69 & 68 \\
\hline Idade média dos entrevistados (anos) & & 47 & 53 & 36 \\
\hline \multirow{3}{*}{ Estado civil (\%) } & Casado & 55,5 & 49 & 52 \\
\hline & Solteiro & 22,2 & 30,5 & 36 \\
\hline & Viúvo e outros & 22,3 & 16 & 8 \\
\hline Tempo médio de moradia (anos) & & 14,9 & 20,5 & 12,1 \\
\hline \multirow{2}{*}{ Tempo de Residência } & $<10$ anos & 42,6 & 16,4 & 44 \\
\hline & $>10$ anos & 57,4 & 83,6 & 56 \\
\hline \multirow{4}{*}{ Escolaridade (\%) } & Não alfabetizados & 13,2 & 16 & 4 \\
\hline & Fundamental & 36,8 & 49 & 36 \\
\hline & Médio completo & 42,6 & 28 & 56 \\
\hline & Superior completo & 7,4 & 7,0 & 4 \\
\hline \multirow{3}{*}{ Origem (\%) } & Acreanos & 79,6 & 79,6 & 88 \\
\hline & Nascidos em Rio Branco & 55,8 & 45,5 & 45,5 \\
\hline & Emigrados do interior do Acre & 44,2 & 54,5 & 54,5 \\
\hline \multirow{3}{*}{ Ocupação (\%) } & Aposentados & 33,0 & 41,8 & 20 \\
\hline & Doméstica & 19,5 & 18,2 & 24 \\
\hline & Outros & 44,5 & 40 & 56 \\
\hline
\end{tabular}

foram encontradas correlações não paramétricas (Spearman) significativas entre riqueza de plantas alimentares e as variáveis quantitativas tempo de moradia $(\mathrm{p}=0,4541)$ e idade dos entrevistados $(\mathrm{p}=0,4028)$.

Estes resultados sugerem que os fatores sócios econômicos analisados não apresentam correlação direta com a riqueza de plantas alimentares cultivadas nos quintais urbanos estudados. Os moradores possuem um bom conhecimento tradicional acerca das plantas que cultivam notando-se, entretanto que este não está diretamente relacionado ao nível de escolaridade dos entrevistados.

A análise de variância não paramétrica para a variável riqueza de plantas alimentares mostrou que existem diferenças significativas entre bairros $\left(p>\chi^{2}=0,0013\right)$ para este fator. $O$ resultado para o bairro Placas ( 8,3 espécies) difere significativamente de Aeroporto Velho (4,6 espécies; $\mathrm{p}>\mathrm{KSa}$ $=0.0095)$ e Novo Horizonte (3,9 espécies; $\mathrm{p}>\mathrm{KSa}=0,008)$ pelo teste de Kolmogorov-Smirnov $(\mathrm{p}<0,05)$, enquanto a comparação entre os bairros Aeroporto Velho e Novo Horizonte não diferem significativamente $(\mathrm{p}>\mathrm{KSa}=0,9696)$.

O bairro Placas apresentou a maior riqueza de espécies frutíferas e arbóreas devido em parte pela maior área física dos quintais. Este fato revela que quintais com áreas maiores apresentam potencial para uso agrícola com espécies frutíferas, sobretudo para fins alimentares quando cultivadas em consórcio com pequenos animais, conforme observado nas entrevistas. O mesmo fato foi constatado em estudos realizado em quintais caseiros de Boa Vista, RR, por Semedo \& Barbosa (2007), que segue um padrão que concentra a escolha de cultivo em espécies frutíferas consagradas por seu êxito na produção de frutos.
A exemplo do bairro Placas, o Novo Horizonte apresentou uma maior concentração de frutíferas, devido também ao maior espaço disponível nos quintais. Estes dados corroboram os estudos de Lamont et al. (1999) que reportaram, no Pará, que quanto maior o tamanho do lote, maior era a riqueza de espécies. No entanto, esta relação não foi observada nos estudos realizados por Albuquerque et al. (2005) e Eichemberg et al. (2009).

Os quintais do bairro Placas, segundo o seu tipo de organização espacial e arranjo dos vegetais, se assemelham aos sistemas agroflorestais rurais, sem regras de espaço e alinhamento entre as plantas; no entanto, se observou certa setorização na escolha do local de plantio com espécies ornamentais dispostas na frente da residência e as alimentares alocadas nos fundos do quintal. Semelhante distribuição espacial de espécies em quintais foi relatada nos trabalhos de Semedo \& Barbosa (2007) e Amaral \& Guarim Neto (2008).

O bairro Aeroporto Velho apresentou maior área construída e menor área efetiva de quintal. Essa diferença em relação aos demais bairros ocorre devido ao fato dos entrevistados terem um maior tempo médio de moradia (20 anos) no bairro e a maioria destes ( $83 \%$ ) viverem na mesma residência há mais de dez anos (Tabela 2 ). Conseqüentemente, implementam um maior número de benfeitorias com o passar dos anos. De acordo com Coomes \& Ban (2004), o tamanho do quintal varia em função da condição econômica da família, modo de propagação das plantas e do tempo que o morador ocupa o espaço, acrescentando as construções de acordo com suas necessidades.

Em relação à conservação da agrobiodiversidade, os quintais urbanos são considerados um verdadeiro banco de 
recursos genéticos de grande importância para a humanidade. $\mathrm{Na}$ Amazônia são cultivadas espécies alimentares, medicinais e condimentares em quintais urbanos e rurais, geralmente localizados próximos à cozinha, o que facilita os cuidados. As mulheres são as responsáveis pela preservação da agrobiodiversidade, pelo cultivo diversificado de espécies contribuindo para a domesticação no quintal de cultivares tradicionais e plantas oriundas de floresta (Murrieta \& Winklerprins 2003).

Os quintais são construídos com uso de insumos externos mínimos e utilizam método sucessional de espécies, preservando parte de plantas nativas, imitando ambientes da floresta. Assim, os quintais urbanos de Rio Branco se constituem uma rica fonte de recursos genéticos vegetais contribuindo com a segurança alimentar, saúde das famílias e eventualmente com geração de renda com a venda dos excedentes não são apenas lugares de lazer e trabalho, mas, representam importante área de estudo aos etnobotânicos como reportado também por Vogt et al. (2004). Os quintais urbanos de Rio Branco são locais de grande diversidade de espécies vegetais ainda que apresentem baixa similaridade florística, variando em sua composição conforme a necessidade dos moradores asemelhando-se com os quintais agroflorestais estudados no Pará, por Ferreira \& Pires Sablayrolles (2009).

Em todos os quintais foi observado que o uso de espécies para alimentação tem papel importante na complementação da dieta alimentar. A manutenção do quintal na residência possui valores intangíveis e difíceis de serem mensurados, como o prazer de cultivar, espaço de lazer, bem estar proporcionado pela melhoria da ambiência (sombra) e da paisagem proporcionada pelas espécies arbóreas. Esta pesquisa pode servir de subsídio para formulação de políticas públicas de saúde pública, segurança alimentar, conservação de recursos genéticos e geração de renda por meio da agricultura urbana.

\section{Referências bibliográficas}

Acre. 2006. Governo do Estado do Acre. Programa Estadual de Zoneamento Ecológico-Econômico. Zoneamento EcológicoEconômico do Acre Fase II Documento síntese - escala 1:250.000. Rio Branco, Secretaria de Estado de Meio Ambiente.

Albuquerque, U.P.; Andrade, L.H.C. \& Caballero, J. 2005. Structure and floristics of homegardens in Northeastern Brazil. Journal of Arid Environments 62: 491-506.

Albuquerque, U.P. \& Lucena, R.F.P. 2004. Métodos e técnicas em na pesquisa etnobotânica. Recife, Livro Rápido/NUPEEA.

Amaral, C.N. \& Guarim Neto, G. 2008. Os quintais como espaços de conservação e cultivo de alimentos: um estudo na cidade de Rosário do Oeste (Mato Grosso, Brasil). Boletim do Museu Paraense Emílio Goeldi. Ciências Humanas 3: 329-341.

Amorozo, M.C.M. 2002. Agricultura tradicional, espaços de resistência e o prazer de plantar. Pp.123-131. In: Albuquerque, U.P.; Alves, A.G.C.; Borges, A.C.L.; Silva, V.A. (Orgs.). Atualidades em etnobiologia e etnoecologia. Recife, SBEE.

Aquino, A.M. \& Assis, R.L. 2007. Agricultura orgânica em áreas urbanas e periurbanas com base na agroecologia. Ambiente \& Sociedade 10: 137-50.

Carniello, M.A.; Silva, R.S.; Cruz, M.A.B. \& Guarim Neto, G. Quintais urbanos de Mirassol D’Oeste-MT, Brasil: uma abordagem etnobotânica. Acta Amazônica 3: 451-470.

Carvalho, G.H.; Cianciaruso, M.V. \& Batalha, M.A. 2010. Plantminer: a web tool for checking and gathering plant species taxonomic information 25: 815-816. http://www.plantminer.com. Environmental Modelling and Software. (Acesso em 22/12/2010).

Coomes, O.T. \& Ban, N. 2004. Cultivated plant species diversity in home gardens of an Amazonian peasant village in Northeastern Peru. Economic Botany 58: 420-34.

Eichemberg, M.T.; Amorozo, M.C.M. \& Moura, L.C. de. 2009. Species composition and plant use in old urban homegardens in Rio Claro, Southeast of Brazil. Acta Botanica Brasilica 23: 1057-75.

Delunardo, T.A. 2008. Agrobiodiversidade em quintais urbanos de Rio Branco. Dissertação (Mestrado, Produção Vegetal), Universidade Federal do Acre, Rio Branco.

Emperaire, L. \& Eloy, L. 2008. A cidade, um foco de diversidade agrícola no Rio Negro (Amazonas, Brasil). Boletim do Museu Paraense Emílio Goeldi. Ciências Humanas 3: 195-211.

Ferreira, T.B. \& Pires Sablayrolles, M.G. 2009. Quintais agroflorestais como fontes de saúde: plantas medicinais na Comunidade de Vila Franca, Reserva Extrativista Tapajós-Arapiuns, Pará. Revista Brasileira de Agroecologia 4: 3159-3162.

Galluzzi, G.; Eyzaguirre, P. \& Negri, V. 2010. Home gardens: neglected hotspots of agro-biodiversity and cultural diversity. Biodiversity and Conservation 19: 3635-3654.

Kumar, B.M. \& Nair, P.K.R. 2004. The enigma of tropical homegardens. Agroforestry Systems 61: 135-152.

Lamont, S.R.; Eshbaugh, W.H. \& Greenberg, A. M. 1999. Species composition, diversity, and use of homegardens among three Amazonian villages. Economic Botany 53: 312-26.

Millat-e-Mustafa, M.D. 1998. An approach towards analysis of homegardens. Pp. 39-48. In: Rastogi, A.; Godble, A. \& Shengji, P. (Eds.). Applied Ethnobotany in natural resource management traditional home gardens. Kathmandu, International Centre for Integrated Mountain Development Kathmandu.

MOBOT, 2010. Missouri Garden W3 tropicos. Disponível em: $<$ http:// www.mobot.mobot.org>. (Acesso em: 05/11/2010).

Murrieta, R.S.S. \& WinklerPrins, A.M.G.A. 2003. Flowers of water: homegardens and gender roles in a riverine caboclo community in the lower Amazon, Brazil. Culture and Agriculture 25: 35-47.

Nair, P.R. 2004. The enigma of tropical homegardens. Agroforestry Systems 12: 135-152.

Oliveira, L.A.P. 1982. O sertanejo, o brabo e o posseiro: A periferia de Rio Branco e os cem anos de andança da população acreana. Belo Horizonte, Universidade Federal de Minas Gerais.

Rio Branco. 2007. Levantamento e mapeamento de solos do município de Rio Branco, AC, na escala de 1:100.000. Rio Branco, Prefeitura Municipal de Rio Branco.

SAS. SAS Institute Inc ${ }^{\circledR}$ 2003. Cary, NC, USA, Lic. UDESC: SAS Institute Inc, 2003.

Schmink, M. \& Cordeiro, M.L. 2008. Rio Branco: A cidade da florestania. Belém, EDUFPA. Editora da Universidade Federal do Pará.

Semedo, R.J.C.G. \& Barbosa, R.I. 2007. Árvores frutíferas nos quintais urbanos de Boa Vista, Roraima, Amazônia brasileira. Acta Amazônica 37: 561-568.

Singer, P. 2002. Introdução à Economia Solidária. São Paulo, Fundação Perseu Abramo.

Slinger V.A. 2000. Peri-urban agroforestry in the Brazilian Amazon. The Geographical Review 90: 177-90.

The Angiosperm Phylogeny Group. 2009. An update of the Angiosperm Phylogeny Group classification for the orders and families of flowering plants: APG III. Botanical Journal of the Linnean Society 161: 105-121.

Vogl, C.R.; Vogl-Lukasser, B. \& Puri, R.K. 2004. Tools and methods for data collection in ethnobotanical studies of homegardens. Field Methods 16: 285-306,

Winklerprins, A.M.G.A. \& Sousa, P.S. 2005. Surviving the city: Urban homegardens and the economy of affection in the Brazilian Amazon. Journal of Latin American Geography 4: 103-122. 